\title{
X-linked intellectual disability-retinitis pigmentosa syndrome
}

INSERM

\section{Source}

INSERM. (1999). Orphanet: an online rare disease and orphan drug data base. $\underline{X \text {-linked }}$ intellectual disability-retinitis pigmentosa syndrome. ORPHA:85332

X-linked intellectual disability-retinitis pigmentosa syndrome is characterized by moderate intellectual deficit and severe, early-onset retinitis pigmentosa. It has been described in five males spanning three generations of one family. Some patients also had microcephaly. It is transmitted as an X-linked recessive trait. 